\title{
Es ist besser, Fremdsprachen im Zielsprachenland zu lernen - Lernerauffassungen hinsichtlich der Rolle von Auslandsaufenthalten beim Fremdsprachenlernen
}

\author{
It is better to learn a foreign language in the foreign \\ country - learner beliefs \\ about the role of study abroad
}

\begin{abstract}
Study abroad contexts are assumed by foreign language learners to be the best environment for effective language learning. This article reports on beliefs of first year Polish students of German studies. The collected qualitative data show that Polish students do not differ from other learners with regard to their opinions on study abroad and its benefits (and disadvantages) for speaking German. The article underlines the need to reflect with learners about their beliefs, experiences, and expectations of language learning.
\end{abstract}

KEYWORDS: learner beliefs, speaking skill, study abroad, diary studies.

\section{VORÜBERLEGUNGEN}

Fast alle Lernenden ( $=$ L.) sind der Auffassung, dass man Fremdsprachen am leichtesten im Zielsprachenland (= ZSL) lerne. Davon zeugen die Ergebnisse zahlreicher quantitativer und qualitativer Studien zu den learner beliefs about language learning, die seit den 1980er Jahren durchgeführt werden (vgl. exemplarisch Horwitz 1988: 288, Yang 1999: 523, Mercer 2010: 441). Diese Auffassung vertreten auch die meisten Lehrenden. Ein kurzer oder längerer Auslandsaufenthalt wird bildlich als Eintauchen in die Fremdsprache dargestellt (vgl. Appel 2000: 165ff.). Die mit einem Auslandsaufenthalt verbundenen Erwartungen der L. und Lehrenden beziehen sich vor allem auf die Verbesserung der Sprechkompetenz. 
Ziel dieses Aufsatzes ist es zu diskutieren, wie polnische Germanistikstudenten (=GS) ihre Auffassungen (= A.) in dieser Hinsicht begründen und in welchem Zusammenhang diese zu deren schulischen Fremdsprachenlernerfahrungen stehen. Fokussiert werden vor allem die A., die sich auf die Entwicklung der Sprechkompetenz beziehen. Darüber hinaus wird überlegt, welche Auswirkungen die von den GS vertretenen A. auf die Lernund Kommunikationsprozesse im Studium haben können. Der Beitrag wird mit einigen didaktischen Empfehlungen und einem Ausblick auf Nachfolgeuntersuchungen abgeschlossen.

\section{THEORETISCH-EMPIRISCHER HINTERGRUND}

Das Forschungsinteresse an der Rolle der Auslandsaufenthalte beim Fremdsprachenlernen (= FL) reicht in die späten 1960er Jahre zurück. Die seitdem durchgeführten Untersuchungen lassen sich nach Freed (1998: 3342) in zwei Gruppen einteilen:

\section{1) Test-Based Studies}

Im Mittelpunkt des Forschungsinteresses standen die Sprachproduktionen der L. Mithilfe der Prä- und Posttests wurde erforscht, ob und in welchem Grade sich die Sprechkompetenz während des Auslandsaufenthalts verändert hatte. Seit den 1980er Jahren wurde dabei das Oral Proficiency Interview (=OPI) eingesetzt. In den meisten Untersuchungen konnte zwar nachgewiesen werden, dass die Sprechkompetenz der L. gestiegen war, hinterfragen sollte man aber die Aussagekraft des verwendeten Messinstruments. Mit dem OPI konnte nämlich nicht festgestellt werden, wie der Auslandsaufenthalt die Erhöhung der Sprechkompetenz bedingte (vgl. Freed 1998: 35; Dewaele 2005: 370).

\section{2) Enhanced Format Studies}

Das Ziel dieser Untersuchungen, die sowohl quantitativ als auch qualitativ angelegt waren, bestand darin, die (Nach-)Wirkungen eines Auslandsaufenthalts im Geflecht unterschiedlicher individueller Merkmale der L. (z. B. Geschlecht, Persönlichkeit, Sprachlerneignung etc.) sowie umfeldspezifischer Variablen (z. B. Qualität des Inputs) in den Blick zu nehmen. Die untersuchten Sprachlernbiographien wurden daher individuell analysiert. Charakteristisch waren dabei vergleichende Studien, in denen die Leistungen der L., die aus dem ZSL zurückgekehrt waren, mit den Leistungen ihrer Kommilitonen, die im Herkunftsland (= HL) die Sprache lernten, verglichen wurden (nach Freed 1998: 43ff.). Eine besondere Bedeutung kam den Untersuchungen $\mathrm{zu}$, in denen die Entwicklung soziolinguistischer Kompetenz 
erforscht wurde (vgl. Marriott 1995). Darüber hinaus spielten die A. eine wichtige Rolle, weil sie in direkter Weise Einsicht in die Auslandserfahrungen der L. gewährten (vgl. Miller, Ginsberg 1995).

Im Folgenden werden die wichtigsten Ergebnisse der bisherigen Untersuchungen zusammengeführt:

1) Ein Auslandsaufenthalt ist für die Entwicklung der Sprechkompetenz von Vorteil. Die L. sprechen in der Regel fließender, machen kürzere Pausen zwischen einzelnen Äußerungen, gebrauchen in ihrem Input mehr Modifizierungen, Formelsequenzen und Kompensationsstrategien, begehen dagegen nach wie vor Fehler (vgl. Freed, So, Lazar 2003).

2) Die L. profitieren von einem Auslandsaufenthalt besonders dann, wenn sie über solide Grundlagen in Grammatik und Leseverstehen verfügen, die im HL entwickelt wurden. Effektiver ist dagegen im ZSL der Wortschatzerwerb (vgl. Collentine 2004: 245).

3) Wilkinson (1997, zit. nach Pellegrino 1998: 97) widerlegt den so genannten Sprachmythos (language myth), nach dem ein Auslandsaufenthalt den Gebrauch der Zielsprache begünstige oder sogar erzwinge. Wilkinson ist im Gegensatz zu DeKeyser (1991) nicht der Meinung, dass manche L. aus Faulheit (lazy student myth) den Gebrauch der Zielsprache vermeiden würden. Es gebe mehrere andere Gründe, weswegen die L. die Vermeidungsstrategien anwenden. Hierzu zählen: Probleme mit Hörverstehen, geringe Risikobereitschaft, traditionelle Einstellungen der grammatischen Korrektheit gegenüber, Persönlichkeitsmerkmale, schließlich Ziele und Erwartungen, die die L. mit einem Auslandsaufenthalt verbinden (vgl. Pellegrino 1998: 98). Häufig wird bei multikulturellen Begegnungen oder unterschiedlichen Sprachniveaus die englische Sprache benutzt (vgl. Ehrenreich 2008: 110, hierzu auch Churchill 2006: 226). Code-switching wird von den L. als „Anzeichen der fortschreitenden Integration in die Zielkultur - als ein Dazwischen-Sein" - angesehen (vgl. Perrefort 2008: 64). Perrefort (2008: 66) konstatiert:

Der häufig geäußerte Wunsch, die Sprache der anderen wie ein Muttersprachler zu sprechen, um als Mitglied der Gruppe angesehen zu werden, verleiht dem Bedürfnis Ausdruck, nach einer gewissen Zeit wieder so zu sein wie alle anderen und nicht mehr aufzufallen.

4) Die L. äußern sich durchaus kritisch über ihren schulischen Fremdsprachenunterricht $(=\mathrm{FU})$. Beim Sprachgebrauch außerhalb der Klasse wenden sie jedoch häufig die institutionellen Interaktionsmuster an und haben für den FU charakteristische Erwartungen (z. B. Feedback und Korrekturen seitens der native speakers [= NS]) (vgl. Wilkinson 1998b: 25). 
5) Umstritten ist die Rolle der Gastfamilien und der ganzen zielsprachlichen Umgebung. Wilkinson (1997, zit. nach Pellegrino 1998: 110, vgl. auch Wilkinson 1998a: 133) stellt den so genannten homestay myth in Frage, nach dem die zielsprachliche Umgebung die Entwicklung der Sprechkompetenz immer fördern solle. Dies ist von der Qualität des Inputs abhängig. Einerseits werden die sprachlichen Kontakte in den Gastfamilien nur auf bestimmte Themenbereiche eingeschränkt (vgl. O'Donnel 2004: 88), andererseits können sich manche L. wegen der Fülle und der Qualität des Inputs überfordert fühlen und jegliche Kontakte zu NS vermeiden (Segalowitz, Freed 2004: 174; Dewaele 2005: 369). ${ }^{1}$ Außerdem spielen das Geschlecht und die Persönlichkeitsmerkmale der NS eine wichtige Rolle (vgl. Pellegrino Aveni 2005: 55). Pellegrino (1998: 109) betont, dass die L. von der zielsprachlichen Umgebung die Gewährleistung sozial-psychologischer Sicherheit erwarten.

6) Schwierigkeiten bereiten den L. kulturelle Unterschiede, die zu Übergeneralisierungen oder Missverständnissen führen können (vgl. Freed 1998: 50). Fortgeschrittene L. eignen sich sozio- und pragmalinguistische Aspekte leichter an als Anfänger (vgl. Kasper 1996; nach Regan 1998: 73f.).

7) Bei den Anfängern sind dagegen die Fortschritte in der Kompetenzentwicklung häufiger bemerkbar (vgl. Adams 2006: 260).

8) Kurze Auslandsaufenthalte sind manchmal effektiver als lange (vgl. Dwyer 2004: 155).

9) Heterogene Forschungsergebnisse lassen sich bezüglich der Geschlechtsrolle bei einem Auslandsaufenthalt feststellen. Während die einen Studien (z. B. Davidson 2010: 20) auf keine signifikanten Unterschiede hinweisen, wird in den anderen Untersuchungen behauptet, dass Männer risikobereiter als Frauen seien und häufiger Kontakte zu Fremden herstellen würden (vgl. Brecht, Davidson, Ginsberg 1993: 16; zit. nach Freed 1998: 37; auch Polanyi 1995).

10) Für L., die eine Fremdsprache im ZSL lernen (möchten), sind perfektionistische Ansprüche und hohe Erwartungshaltungen kennzeichnend (vgl. Perrefort 2008: 60).

Viele seit den 1990er Jahren geführte Untersuchungen sind Einzelfallanalysen, bei denen introspektive Verfahren angewendet werden. Eine Generalisierung der Ergebnisse ist nur bedingt möglich. Den Forschenden geht es vielmehr darum, individuelle Erfahrungen und A. der L. kennenzulernen als weitgehende Schlussfolgerungen zu ziehen. Pellegrino (1998: 93) betont:

${ }^{1}$ Nach wie vor fehlen breit angelegte Untersuchungen über kausale Zusammenhänge zwischen der Qualität des zielsprachlichen Inputs und den mündlichen Produktionen der L. (vgl. Martinsen 2008: 507). 
Students' perceptions of the language, their own learning, and the study abroad experience hold great value for understanding the language use and social behaviors of students immersed in an L2 environment.

Darüber hinaus gibt es wenige institutionelle Empfehlungen bezüglich dessen, was L. im Ausland erwerben/lernen sollten (vgl. Ehrenreich 2008: 108). Das von Lambert (1993: 315; zit. nach O’Donnell 2004: 4) geäußerte Postulat hat daher an seiner Aktualität nichts eingebüßt:

(...) We don't know, when is the best time to send students, what kind of students can profit most from language study abroad, how much and what kinds of language learning they should hale before they go, or how best to manager the mix of informal and extra-classroom learning, and what happens inside the classroom. We don't even know what language gains the students actually make.

\section{BESCHREIBUNG DER UNTERSUCHUNG}

Im Folgenden werden die A. polnischer GS hinsichtlich der Rolle eines Aufenthalts in einem deutschsprachigen Land dargelegt, unter besonderer Berücksichtigung der Sprechfertigkeit. An der Untersuchung nahmen 83 GS (73 Frauen und 10 Männer) von der Universität Szczecin (3. Semester) und von der Technischen Universität Koszalin (1. Semester) teil.. ${ }^{2}$ Nur wenige GS hatten längere private Aufenthalte in einem deutschsprachigen Land hinter sich, relativ viele (besonders im 1 . Semester) waren dagegen noch nie in einem deutschsprachigen Land gewesen.

Als Instrument der Datenerhebung dienten dialogische Lerntagebücher (vgl. hierzu Nerlicki 2009). Die Aufgabe der GS bestand darin, zu ausgewählten Problemen des FL schriftlich Stellung zu nehmen. Die Lerntagebücher wurden dann von dem Autor eingesammelt, gelesen und kommentiert, so dass es zu einem Meinungsaustausch kam. Um den GS die Stellungnahme zu dem behandelten Forschungsproblem zu erleichtern, wurde eine allgemeine Leitfrage formuliert: Kann man Ihrer Ansicht nach schneller sprechen lernen, wenn man in einem deutschsprachigen Land lebt oder sich dort einige Zeit aufhält? Falls ja/nein: Warum? Die GS konnten ihre A. in deutscher oder polnischer Sprache niederschreiben. ${ }^{3}$ Die Analyse erfolgte nach den Prinzipien der explorativ-interpretativen Methodologie.

2 Diese Untersuchung hatte nicht zum Ziel, eventuelle Unterschiede zwischen den A. der Studienanfänger in der Germanistik (1. Semester) und denen der GS aus dem 3. Semester herauszustellen.

${ }^{3}$ Die auf Polnisch verfassten Lerntagebucheinträge wurden ins Deutsche übersetzt. 


\section{ERGEBNISSE}

Die durchgeführte Untersuchung lässt darauf schließen, dass polnische GS typische A. hinsichtlich der Rolle eines Auslandsaufenthalts bei der Entwicklung fremdsprachlicher Sprechkompetenz vertreten. Im Folgenden wird auf die wichtigsten A. eingegangen. Einige von ihnen werden mit Ankerbeispielen belegt.

1) Durch die meisten Lerntagebucheinträge zieht sich der Gedanke, dass es im ZSL mehr Sprechgelegenheiten gebe, die sich per se aus diversen Kommunikationssituationen ergeben. Die GS sind der Ansicht, dass man der deutschen Sprache ausgesetzt werde und dass es nicht möglich sei - so im Sinne von Watzlawick - nicht zu kommunizieren. Die Involvierung in eine Kommunikationssituation erzwinge gewissermaßen den Sprachgebrauch. Dies veranschaulicht das folgende Beispiel:

(1) Diejenigen, die Deutsch in Polen lernen, vermeiden oft zu sprechen. Ich weiß es aus eigener Erfahrung. Es fällt manchmal schwer, sich zu überwinden. [...] Wenn wir in fremder Umgebung sind, erzwingt sie von uns, die Sprache zu gebrauchen.

2) Das vor Ort gesprochene Deutsch wird im Gegensatz zum institutionell gelernten Schuldeutsch als eine natürlichere Sprachform aufgefasst. "Trockenem“ Grammatiklernen im FU wird der Gebrauch der Umgangssprache im ZSL entgegengesetzt. Nicht selten wird diese Opposition als künstliches vs. natürliches Deutschlernen bezeichnet.

3) Die GS bemerken, dass die von den NS verwendete gesprochene Umgangssprache von der im Deutschunterricht (=DU) vermittelten Standardsprache abweiche, ganz zu schweigen davon, dass sie nicht immer korrekt sei. Laut GS sind die auftretenden sprachlichen Unzulänglichkeiten seitens der NS sowie der L. ganz natürlich, weil man sich sowieso verständigen kann. Den Lerntagebucheinträgen ist zu entnehmen, dass die im DU gewonnenen theoretischen Sprachkenntnisse für die Entwicklung der Sprechkompetenz nicht unentbehrlich sind. Sie erweisen sich dagegen als nützlich, wenn die Regeln im DU bewusst nachvollzogen werden (sollen):

(2) Ich habe Deutsch in Deutschland gelernt. Nach der Rückkehr nach Polen wurde ich einmal in der Schule gebeten zu erklären, warum das Substantiv Präsident im Genitiv die Endung -en hat. Ich konnte es nicht. Ich habe dem Lehrer gesagt, dass diese Endung einfach hineinpasst. Eine theoretische Unterstützung wäre dabei nützlich gewesen.

Das obige Beispiel zeigt, dass der institutionelle DU im HL mit einer verstärkten Fokussierung der Regelkenntnisse assoziiert wird. Die Entwicklung 
der Sprechkompetenz erfordere dagegen einen aktiven Umgang mit der Sprache, der dem DU offensichtlich nach wie vor fehle.

4) Die GS vergleichen das Lernen einer Fremdsprache im ZSL mit dem L1-Erwerb im Kindesalter, indem sie auf natürliche Bedingungen solch eines Prozesses hinweisen:

(3) In Deutschland kann man beobachten, wie die Einheimischen sprechen. Ich glaube, solch eine Lernweise ist interessanter und nützlicher. Es ist so wie bei Kindern, wenn sie z. B. die Namen von Bäumen kennenlernen. Natürlich werden sie diese auch lernen, wenn sie nur Bilder sehen. Aber schneller beherrschen die Kinder diese Namen, wenn wir sie in den Park nehmen. Dann können sie vor Ort die Bezeichnungen mit den Bäumen vergleichen.

5) In vielen Lerntagebucheinträgen wiederholen sich die A., dass ein Auslandsaufenthalt den GS ihre Sprechangst abbauen helfe. Hierzu das folgende Beispiel:

(4) Als ich in der deutschen Umgebung verweilt habe, musste ich Polnisch vergessen, weil niemand die Sprache verstanden hat. Ich habe mich auf meine Sprachfähigkeiten verlassen, auf meinen reichen Wortschatz, auf meine Grammatikkenntnisse, aber vor allem auf mein positives Denken. [...] Zurzeit kann ich sagen, dass ich meine größten Probleme gelöst habe. Ich habe keine Sprechangst mehr. Wenn ich etwas nicht weiß oder nicht verstehe, frage ich ganz direkt nach einer Erklärung.

Die GS, deren Selbstwert- und Selbstwirksamkeitsgefühl verhältnismäßig niedrig sind, werden nach einem Auslandsaufenthalt selbstbewusster und risikobereiter.

6) Dem obigen Beispiel ist außerdem zu entnehmen, dass der Sprachgebrauch im Ausland das Denken auf Polnisch zugunsten des Denkens auf Deutsch vermindere. Die GS begründen häufig ihre Sprechprobleme damit, dass die L1 beim Gebrauch der deutschen Sprache eingeschaltet werde und die Produktion einer Äußerung beeinträchtige, so dass diese Äußerung allzu Polnisch klinge. Die GS kommentieren dies folgendermaßen:

(5) Als ich in Deutschland an einem Sprachkurs teilnahm, gab es keine Polen in der Gruppe. [...] Ich musste mein Denken „auf Polnisch“ ausschalten und deswegen machte ich weniger Fehler und konnte mehr Wörter und Wendungen lernen. [...] Wenn man in der Klasse Deutsch spricht, steckt die polnische Sprache irgendwie im Hinterkopf. Sie kann auf den Gebrauch des Deutschen negativ einwirken.

(6) Denken auf Deutsch bedeutet für mich, die Sätze sofort in der deutschen Sprache zu bilden. [...] Wenn man auf Deutsch denken möchte, sollte man in diesem Moment die polnische Sprache gänzlich vergessen, die polnischen Re- 
geln und die Grammatik beiseite legen und auf die deutsche Grammatik umschalten.

Die GS erhoffen sich von einem Auslandsaufenthalt, ihre mündlichen Äußerungen fließender zu produzieren, wobei damit in der Regel schneller gemeint ist.

7) Diejenigen GS, die schon einmal in einem deutschsprachigen Land gewesen sind, sind sich dessen bewusst, dass solch ein Auslandsaufenthalt, besonders beim ersten Mal, unterschiedliche Verständnisprobleme impliziert, die per se das Sprechen erschweren.

8) Die A. derjenigen GS, die in einem deutschsprachigen Land noch nie gewesen sind, basieren auf A. und Erfahrungen anderer L. oder fungieren als typische Annahmen hinsichtlich des FL. Dies verdeutlicht das folgende Beispiel:

(7) Ich glaube, in Deutschland kann man Deutsch schneller lernen. Solche Lernweise erfordert weniger Aufwand, wir lernen einfach unwillkürlich. Ich habe gehört, dass man in einem Jahr die Sprache unbewusst beherrschen kann. Ich weiß nicht, inwieweit dies wahr ist. Trotzdem bin ich der Meinung, dass die Personen, die sich einige Zeit in Deutschland aufhalten, leichter die Sprache erlernen können.

[Lehrer:] Was meinen Sie mit der Bezeichnung die Sprache unbewusst beherrschen?

Es geht mir um die Regelkenntnisse. Man lernt die Regeln unbewusst.

[Lehrer:] Woher wissen Sie das?

Ich kenne Personen, die die Sprache wirklich in einem Jahr beherrscht haben. Sie meinen, sie hätten in Deutschland viel mehr als in der Schule gelernt. Ich würde gerne dies ausprobieren.

Dass die GS allzu große Erwartungen mit einem Auslandsaufenthalt verbinden, zeugen die A., dass man im Ausland unwillkürlich lerne und dies weniger Aufwand erfordere. Es scheint, dass die GS - wie bereits bemerkt wurde - den im FU aufzubringenden Aufwand vor allem mit den bewusst anzueignenden Sprachregeln verbinden. Die Dichotomie bewusst vs. unbewusst wird in den A. der GS grundsätzlich mit der Form der Sprachaneignung im Sinne von Lernen vs. Erwerben in Zusammenhang gebracht.

\section{SCHLUSSFOLGERUNGEN UND DIDAKTISCHE EMPFEHLUNGEN}

Die Analyse erlaubt es, folgende Schlussfolgerungen zu ziehen:

1) Die polnischen GS unterscheiden sich kaum in ihren A. von anderen L. Demzufolge zählt die Auffassung, dass das FL im ZSL förderlich sei, zu den typischen A., die von den zu lernenden Fremdsprachen, dem gesellschaftlichen Lernhintergrund, den individuellen Lernerfahrungen und Per- 
sönlichkeitsmerkmalen eher unabhängig sind. Auffallend ist, dass sich die GS, die längere Auslandsaufenthalte hinter sich haben, auch gewisser Schwierigkeiten sowie Nachteile bewusst sind.

2) Aus den A. der GS sticht hervor, dass sich die Lernprozesse im ZSL aus natürlichen Kommunikationsanlässen ergeben, die durch ihre starke Verankerung in jeweiligen Kommunikationssituationen effizienter als im DU wirken würden. Die „Natürlichkeit" einer Kommunikationssituation im ZSL besteht darin, dass man in diese - im Gegensatz zum DU im HL - persönlich involviert wird. Daher wird von den GS beiläufiges als intentionales, institutionell gesteuertes Lernen bevorzugt, wobei sie die Vorteile des DU, besonders beim Grammatiklernen, zu schätzen wissen.

3) Der Gebrauch der deutschen Sprache im ZSL wird mit dem kindlichen L1-Erwerb verglichen, so dass sich die GS von einem Auslandsaufenthalt schnelle Erfolge erhoffen. Dies ist nicht verwunderlich, weil die meisten L. meinen, dass Kinder Fremdsprachen leichter als Erwachsene lernen würden (vgl. exemplarisch Horwitz 1988: 287). In Anbetracht der Unterschiede zwischen dem früh- und nachzeitigen Spracherwerb können solche allzu generalisierenden A. negative Auswirkungen auf die Lern- und Kommunikationsprozesse beim institutionellen Deutschlernen haben. Dem können besonders diejenigen GS ausgesetzt werden, die nicht oft die Möglichkeit haben, Deutsch in einem deutschsprachigen Land zu verwenden.

4) Die GS sehen in einem Auslandsaufenthalt die Möglichkeit, durch häufige Kontakte zu NS ihre Sprechangst zu vermindern. Dies ist umso wichtiger, weil die L. nicht selten befürchten, dass NS ihre Fehler erkennen und deswegen ihre Sprechkompetenz niedrig beurteilen würden.

Vor dem Hintergrund der durchgeführten Untersuchung lassen sich einige didaktische Empfehlungen nennen:

1) Da fremdsprachliche Lern- und Kommunikationsprozesse individuell verlaufen und sich nur teilweise aus der Außenperspektive der Forschenden erfassen lassen, spielen die A. der L. in der Fremdsprachendidaktik eine wichtige Rolle. Anzustreben ist, reflexives Deutschlernen der GS zu fördern, indem deren Lern- und Kommunikationsprozesse auf unterschiedliche Art und Weise - individuell und/oder in Seminargruppen, mündlich und/ oder schriftlich - thematisiert werden. Um dies zu gewährleisten, müssen die GS zielstrebig dazu angeleitet werden, zumal das Reflektieren über das eigene Lernen nicht immer leichtfällt, ganz zu schweigen davon, dass wenige GS im schulischen DU darauf vorbereitet wurden.

2) Wichtig ist, die GS auf einen Auslandsaufenthalt (besonders auf ein Teilzeitstudium) gut vorzubereiten. Solch eine Vorbereitung sollte darin bestehen, dass man die GS auf falsche A. oder zumindest übertriebene Erwartungen aufmerksam macht, die nicht selten von anderen L. (oder Lehren- 
den) unreflektiert übernommen werden. Empfehlenswert ist die Entwicklung von Lern- und Kommunikationsstrategien, die den GS den zielsprachlichen Input bewusst fokussieren helfen. Damit hängen die hier exemplarisch genannten Fragen zusammen, die sich die GS während eines Auslandsaufenthalts stellen können: Wie drücken die NS Sachverhalte aus, die mich interessieren (z. B. an der Universität, im Alltag etc.)? Von welchen NS stammt der qualitativ beste Input für mich? Welche Schwierigkeiten habe ich beim Sprechen im ZSL? Wo? Vor wem? Wie gehe ich mit dem Input um, um die eigene Sprechkompetenz zu verbessern?

3) Aus didaktischer und empirischer Perspektive wäre es wichtig kennenzulernen, wie die GS ihre Aufenthalte und deren Wirkungen auf die Entwicklung der Sprechkompetenz nachvollziehen und beurteilen können. Aus diesem Grunde empfiehlt es sich, die GS zur laufenden oder retrospektiven Selbstevaluation (z. B. in Form von Lerntagebüchern, Sprachlernbiographien) zu ermuntern. Durch eine Nachdiskussion im Forum werden die individuellen Erfahrungen der reflektierenden GS und ihrer Kommilitonen, die im HL lernen, vertieft.

4) Da der traditionelle FU im Vergleich mit dem FL im ZSL oft kritisiert wird, sollten die GS, besonders am Anfang des Studiums, eine Zwischenbilanz ihrer bisherigen fremdsprachlichen Lern- und Kommunikationsprozesse machen. Es wäre nützlich, starke und schwache Punkte des institutionellen (schulischen und universitären) Deutschlernens in Polen herauszugreifen und gemeinsam zu überlegen, wie die didaktische Praxis verändert werden könnte.

\section{AUSBLICK AUF NACHFOLGEUNTERSUCHUNGEN}

Das in diesem Beitrag behandelte Problem eröffnet ein breites Feld für weitere Nachfolgeuntersuchungen. An dieser Stelle werden exemplarisch einige Fragen formuliert:

1) Wie verändern sich die A. der GS nach einem Auslandsaufenthalt?

2) Welchen Einfluss auf die Entwicklung der Sprechkompetenz haben unterschiedliche Kontakte zu NS im ZSL (im institutionell organisierten DU vs. privat)?

3) Welche grammatischen Strukturen der deutschen Sprache werden effizienter im ZSL als im DU im HL erworben?

4) Welchen Einfluss auf die Entwicklung der Sprechkompetenz haben Kontakte zu Nichtmuttersprachlern der deutschen Sprache?

5) Gibt es Ähnlichkeiten/Unterschiede in der Entwicklung der Sprechkompetenz bei L. unterschiedlicher Herkunftssprachen? 


\section{LITERATURVERZEICHNIS}

Adams, R., 2006. Language Learning Strategies in the Study Abroad Context. In: DuFon, M.A., Churchill, E. (Hrsg.). Language Learners in Study Abroad Contexts. Clevedon (u.a.): Multilingual Matters, 259-292.

Appel, J., 2000. Erfahrungswissen und Fremdsprachendidaktik. München: LangenscheidtLongman.

Badstübner, T., Ecke, P., 2009. Student Expectations, Motivations, Target Language Use, and Perceived Learning Progress in a Summer Study Abroad Program in Germany. In: Die Unterrichtspraxis / Teaching German 42, 41-49.

Brecht, R., Davidson, D., Ginsberg, R., 1993. Predictors of Foreign Language Gain during Study Abroad. Washington D.C.: National Foreign Language Center.

Churchill, E., 2006. Variability in the Study Abroad Classroom and Learner Competence. In: DuFon, M.A., Churchill, E. (Hrsg.). Language Learners in Study Abroad Contexts. Clevedon (u.a.): Multilingual Matters, 203-227.

Coleman, J.A., 1998. Language Learning and Study Abroad: the European Perspective. In: Frontiers: The Interdisciplinary Journal of Study Abroad 4, 167-203.

Collentine, J., 2004. The Effects of Learning Contexts on Morphosyntactic and Lexical Development. In: Studies in Second Language Acquisition 26, 227-248.

Davidson, D.E., 2010. Study Abroad: When, How Long, and with What Results? New Data from the Russian Front. In: Foreign Language Annals 43, 6-26.

DeKeyser, R.M., 1991. Foreign Language Development during a Semester Abroad. In: Freed, B.F. (Hrsg.). Second Language Acquisition in a Study Abroad Context. Amsterdam (u.a.): Benjamins, 104-119.

Dewaele, J.-M., 2005. Investigating the Psychological and Emotional Dimensions in Instructed Language Learning: Obstacles and Possibilities. In: The Modern Language Journal 89, 367-380.

Dwyer, M.M., 2004. More is Better: The Impact of Study Abroad Program Duration. In: Frontiers: The Interdisciplinary Journal of Study Abroad 10, 151-163.

Ehrenreich, S., 2008. Sprachlernsituation Ausland: Sprachbad-Mythen und Lingua-FrancaRealitäten. In: Ehrenreich, S., Woodman, G., Perrefort, M. (Hrsg.). Auslandsaufenthalte in Schule und Studium. Bestandsaufnahme aus Forschung und Praxis. Münster: Waxmann, 105-121.

Freed, B.F., 1998. An Overview of Issues and Research in Language Learning in a Study Abroad Setting. In: Frontiers: The Interdisciplinary Journal of Study Abroad 4, 31-60.

Freed, B.F., So, S., Lazar, N.A., 2003. Language Learning Abroad: How Do Gains in Written Fluency Compare with Gains in Oral Fluency in French as a Second Language? In: ADFL Bulletin 34, 34-40.

Horwitz, E.K., 1988. The Beliefs about Language Learning of Beginning University Foreign Language Students. In: The Modern Language Journal 72, 283-294.

Kasper, G., 1996. The Development of Pragmatic Competence. In: Kellerman, E., Weltens, B., Bongaerts, T. (Hrsg.). Eurosla 6. A Selection of Papers. Amsterdam: UV Uitgeverij, 103-120.

Lambert, R.D., 1993. International Education and International Competence in The United States. In: European Journal of Education 28, 309-325.

Marriott, H.E., 1995. The Acquisition of Politeness Patterns by Exchange Students in Japan. In: Freed, B.F. (Hrsg.). Second Language Acquisition in a Study Abroad Context. Amsterdam (u.a.): Benjamins, 197-224. 
Martinsen, R.A., 2008. Short-Term Study Abroad: Predicting Changes in Oral Skills. In: Foreign Language Annals 43, 504-530.

Mercer, S., 2010. A Mindset of EFL: Learners' Beliefs about the Role of Natural Talent. In: English Language Teaching Journal 64, 436-444.

Miller, L., Ginsberg, R., 1995. Folklinguistic Theories of Language Learning. In: Freed, B.F. (Hrsg.). Second Language Acquisition in a Study Abroad Context. Amsterdam (u.a.): Benjamins, 293-315.

Nerlicki, K., 2009. Dzienniczki uczących się jako narzędzie badawcze - próba oceny. In: Neofilolog 32, 151-161.

O'Donnell, K., 2004. Student Perceptions of Language Learning in Two Contexts: At Home and Study Abroad. Universität Pittsburgh. Unveröffentlichte Dissertation.

Pellegrino, V.A., 1998. Student Perspectives on Language Learning in a Study Abroad Context. In: Frontiers: The Interdisciplinary Journal of Study Abroad 4, 91-120.

Pellegrino Aveni, V.A., 2005. Study Abroad and Second Language Use: Constructing the Self. Cambridge: University Press.

Perrefort, M., 2008. Sprachliche Fremderfahrung - Auslöser für Mediationskompetenzen? In: Ehrenreich, S., Woodman, G., Perrefort, M. (Hrsg.). Auslandsaufenthalte in Schule und Studium. Bestandsaufnahme aus Forschung und Praxis. Münster: Waxmann, 57-73.

Polanyi, L., 1995. Language Learning and Living Abroad. In: Freed, B.F. (Hrsg.). Second Language Acquisition in a Study Abroad Context. Amsterdam (u.a.): Benjamins, 271-291.

Regan, V., 1998. Sociolinguistics and Language Learning in a Study Abroad Context. In: Frontiers: The Interdisciplinary Journal of Study Abroad 4, 61-90.

Segalowitz, N., Freed, B., 2004. Context, Contact, and Cognition in Oral Fluency Acquisition. Learning Spanish at Home and Study Abroad Contexts. In: Studies in Second Language Acquisition 26, 173-199.

Wilkinson, S., 1997. Separating Fact from Myth: A Qualitative Perspective on Language Learning during Summer Study Abroad. MLA Convention. Toronto. 29. December 1997.

Wilkinson, S., 1998a. On the Nature of Immersion during Study Abroad: Some Participants Perspectives. In: Frontiers: The Interdisciplinary Journal of Study Abroad 4, 121-138.

Wilkinson, S., 1998b. Study Abroad from the Participants' Perspective: A Challenge to Common Beliefs. In: Foreign Language Annals 31, 23-39.

Yang, N.-D., 1999. The Relationship between EFL Learners' Beliefs and Learning Strategy Use. In: System 27, 515-535. 